Research Paper

\title{
Predictors of the survival of patients with chondro- sarcoma of bone and metastatic disease at diagnosis
}

\author{
Zhan Wang, Genlian Chen, Xiaowei Chen, Xin Huang, Meng Liu, Weibo Pan, Xiaobo Yan, Nong Lin ${ }^{\bowtie}$, \\ Zhaoming Ye $\mathrm{e}^{\bowtie}$ \\ Department of Orthopaedics, Centre for Orthopaedic Research, Orthopedics Research Institute of Zhejiang University, The Second Affiliated Hospital, Zhejiang \\ University School of Medicine, 88 Jiefang Road, Hangzhou, Zhejiang 310000, P.R. China \\ $\square$ Corresponding authors: Zhaoming Ye, MD. Director of Orthopedics Research Institute of Zhejiang University; E-mail: yezhaoming@zju.edu.cn, Tel: \\ +8613606501549. Nong Lin, MD; E-mail: linnong@zju.edu.cn \\ (c) Ivyspring International Publisher. This is an open access article distributed under the terms of the Creative Commons Attribution (CC BY-NC) license \\ (https://creativecommons.org/licenses/by-nc/4.0/). See http://ivyspring.com/terms for full terms and conditions.
}

Received: 2018.10.03; Accepted: 2019.04.07; Published: 2019.06.02

\begin{abstract}
Purpose: Chondrosarcoma with metastatic disease has a very poor prognosis. However, the prognosis and potential prognostic factors of patients with primary chondrosarcoma of bone and metastasis at presentation have not been documented because of its rarity. Therefore, we examined the prognosis of this special cohort and identify possible prognostic factors.

Methods: The Surveillance, Epidemiology, and End Results (SEER) program database was used to identify patients with primary chondrosarcoma of bone and metastatic disease at diagnosis from 2000 to 2013. The prognostic analysis was performed using the Kaplan-Meier method and a Cox proportional hazards regression model.

Results: The SEER database contained 264 cases. The overall survival (OS) and cancer-survival specific (CSS) rates of the entire group at 5 years were $28.4 \%$ and $31.2 \%$, respectively. The median OS and CSS were $14.0 \pm$ 2.5 and $17.0 \pm 2.6$ months, respectively. Multivariate analysis revealed that low tumor grade, surgical treatment, tumor size $<10 \mathrm{~cm}$, and first primary tumor were associated with improved OS. Tumor grade, tumor size, and surgery were independent predictors of CSS. Radiation therapy had no effect on either OS or CSS.

Conclusion: Among patients with primary chondrosarcoma of bone and metastasis at presentation, low tumor grade, surgical treatment, tumor size $<10 \mathrm{~cm}$, and first primary tumor predict prolonged survival.
\end{abstract}

Key words: chondrosarcoma; metastasis at presentation; survival; prognostic factor

\section{Introduction}

Chondrosarcoma is the second most common type of primary bone sarcoma and usually occurs in pelvis and long bones [1, 2]. The demographic, prognostic, and outcome data of chondrosarcoma are well documented. The 5-year survival rate for chondrosarcoma is $75.2 \%$, which is much higher than that of osteosarcoma and Ewing sarcoma [3]. Tumor size, grade, stage, local recurrence, metastasis at presentation, systemic treatment, and radiotherapy are all associated with the prognosis of chondrosarcoma [4-7]. Patients with chondrosarcoma and metastasis often have a poor prognosis $[8,9]$. Recently, Nakamura et al. [10]reported poor prognoses in 20 chondrosarcoma patients after pulmonary metastasis, with a 5-year survival rate
45.7\%. They also found that tumor grade, extrapulmonary metastasis, and treatment for lung metastases predicted survival among those patients who developed lung metastases after the initial treatment of primary chondrosarcoma of the extremities. However, the prognosis and risk factors of patients with primary chondrosarcoma of bone and metastatic disease at initial presentation have not been reported. The effects of radiotherapy and chemotherapy on chondrosarcoma are limited and surgical resection is still the mainstay of treatment [4]. However, some studies have reported that radiotherapy is an effective adjuvant treatment and may provide a survival advantage in chondrosarcoma patients $[6,11,12]$. Therefore, this study also assessed 
the prognostic utility of radiotherapy in primary chondrosarcoma of bone and metastasis at presentation.

To obtain deeper insight into primary chondrosarcoma of bone and metastatic disease at diagnosis, we analyzed all patients from 2000 to 2013 in the Surveillance, Epidemiology, and End Results (SEER) program database of the National Cancer Institute. This was a large-scale study of patients with primary chondrosarcoma of bone and metastatic disease at diagnosis that aimed to confirm the predictors of survival.

\section{Materials and Methods}

\section{Patient population}

A total of 264 patients diagnosed with chondrosarcoma of bone and metastatic disease at initial presentation were identified from 2000 to 2013. All patient data were obtained using the case-listing session procedure from the SEER program (www.seer.cancer.gov). This study followed standard guidelines and was approved by the Ethics Committee of the Second Affiliated Hospital of Zhejiang University School of Medicine.

First, the International Classification of Diseases for Oncology, $3^{\text {rd }}$ edition (ICD-O-3) was used to identify patients with primary chondrosarcoma (ICD-O-3 codes 9180, 9220, 9221, 9231, 9240, 9242, 9243), using the case-listing procedure. Only patients with metastatic disease were enrolled, by reference to the tumor stage at diagnosis. Chondrosarcoma arising from the extraskeletal locations was excluded. Patients diagnosed before 2000 year were also excluded. All patient diagnoses were confirmed histologically, based either on biopsy results or the surgical specimen. Sixteen patients diagnosed only on the basis of the clinical presentation, or according to the radiography, autopsy, or death records were excluded. One patient with missing survival information was also excluded. Data extracted from the SEER database included age, gender, year of diagnosis, race, location, tumor grade, tumor type, tumor size, surgical treatment, radiation treatment, tumor sequence, cause of death, and survival time. Treatment for tumors in our study refers to treatment for primary tumors. Tumor sequence refers to the sequence in which primary chondrosarcomas occurred in various primary tumors of one patient. We divided the location into four categories: (1) axial (pelvis and spine), (2) appendicular (long and short bones of the upper and lower extremities), (3) rib, sternum, and clavicle, and (4) other locations (mandible, skull, and other atypical locations).
Table 1. Clinical characteristics of 264 patients with primary chondrosarcoma of bone and metastatic disease at presentation identified in the SEER Program database from 2000 to 2013

\begin{tabular}{|c|c|}
\hline Category & Value \\
\hline Mean age (years) & 57.4 \\
\hline Median age (years) & 58 \\
\hline \multicolumn{2}{|l|}{ Age(years) } \\
\hline$<60$ & $139(52.7 \%)$ \\
\hline$\geq 60$ & $125(47.3 \%)$ \\
\hline \multicolumn{2}{|l|}{ Gender } \\
\hline Female & $100(37.9 \%)$ \\
\hline Male & $164(62.1 \%)$ \\
\hline \multicolumn{2}{|l|}{ Race } \\
\hline White & $228(86.4 \%)$ \\
\hline Other & $36(136.6 \%)$ \\
\hline \multicolumn{2}{|l|}{ Location } \\
\hline Appendicular & $97(36.7 \%)$ \\
\hline Axial & $78(29.5 \%)$ \\
\hline Rib, sternum and clavicle & $46(17.4 \%)$ \\
\hline Other locations & $43(16.3 \%)$ \\
\hline \multicolumn{2}{|l|}{ Tumor grade ${ }^{a}$} \\
\hline Low & $112(42.4 \%)$ \\
\hline High & $79(29.9 \%)$ \\
\hline Unknown & $73(27.7 \%)$ \\
\hline \multicolumn{2}{|l|}{ Tumor type } \\
\hline Chondrosarcoma, not otherwise specified (NOS) & $178(67.4 \%)$ \\
\hline Juxtacortical chondrosarcoma & $2(0.8 \%)$ \\
\hline Myxoid chondrosarcoma & $24(9.1 \%)$ \\
\hline Mesenchymal chondrosarcoma & $10(3.8 \%)$ \\
\hline Clear cell chondrosarcoma & $2(0.8 \%)$ \\
\hline Dedifferentiated chondrosarcoma & $48(18.2 \%)$ \\
\hline \multicolumn{2}{|l|}{ Tumor size } \\
\hline Median (cm) & 10.5 \\
\hline$<10 \mathrm{~cm}$ & $70(26.5 \%)$ \\
\hline$\geq 10 \mathrm{~cm}$ & $87(33.0 \%)$ \\
\hline Unknown & $107(40.5 \%)$ \\
\hline \multicolumn{2}{|l|}{ Surgery } \\
\hline Yes & $172(65.2 \%)$ \\
\hline No & $92(34.8 \%)$ \\
\hline \multicolumn{2}{|l|}{ Radiation treatment } \\
\hline Yes & $90(34.1 \%)$ \\
\hline No & $174(65.9 \%)$ \\
\hline \multicolumn{2}{|l|}{ Treatment modality } \\
\hline Surgery+radiotherapy & $53(20.1 \%)$ \\
\hline Surgery only & $119(45.1 \%)$ \\
\hline Radiotherapy only & $37(14.0 \%)$ \\
\hline No therapy & $55(20.8 \%)$ \\
\hline \multicolumn{2}{|l|}{ First cancer } \\
\hline Yes & $233(88.3 \%)$ \\
\hline No & $31(11.7 \%)$ \\
\hline \multicolumn{2}{|l|}{ Dead } \\
\hline Yes & $194(73.5 \%)$ \\
\hline No & $70(26.5 \%)$ \\
\hline 5-year OS rate & $28.4 \%$ \\
\hline 5-year CSS rate & $31.2 \%$ \\
\hline 10-year OS rate & $22.8 \%$ \\
\hline 10-year CSS rate & $26.6 \%$ \\
\hline
\end{tabular}

\section{Statistical methods}

The statistical analyses were performed using Microsoft Excel 2016 (Microsoft Corp., Redmond, WA, USA) and SPSS software (ver. 21.0; SPSS Inc., Chicago, IL, USA). Overall survival (OS) was defined as the time from diagnosis to death from any cause and cancer-specific survival (CSS) was regarded as the time from diagnosis to death due specifically to 
cancer. The OS or CSS rate and median survival was calculated by life table method. Observations were censored if the patient was alive at the time of the last follow-up. Univariate analyses were performed using the Kaplan-Meier method with the log-rank test. Multivariate analysis was used to determine the independent predictors of OS and CSS with a Cox proportional hazards regression model. The hazard ratios (HRs) and corresponding 95\% confidence intervals (CIs) were used to show the effect of various factors on OS and CSS. Differences were deemed statistically significant if the P-value was less than 0.05 .

\section{Results}

\section{Clinical characteristics of the $\mathbf{2 6 4}$ patients with metastatic chondrosarcoma}

In total, 264 patients were eligible for our study. Age was categorized based on the median age. In terms of location, $36.7 \%$ primary tumors were located in the extremities, $29.5 \%$ in the axial skeleton, and $17.4 \%$ in the rib, sternum or clavicle. Histologically, $42.4 \%$ of the cases were low grade, and $29.9 \%$ were high grade. Information on the tumor size was available in $59.5 \%$ cases, and was categorized based on the median tumor size. More than half of the patients $(65.2 \%)$ received local surgery and 90 patients $(34.1 \%)$ received radiation treatment. The majority of the patients were diagnosed as first malignant primary tumors $(88.3 \%)$. Ultimately, 194 patients (73.5\%) died, of whom 149 died of cancer. The 5- and 10 -year OS rates of the entire cohort were $28.4 \%$ and $22.8 \%$, respectively. The 5- and 10-year CSS rates were $31.2 \%$ and $26.6 \%$, respectively (Table 1 ). The median OS and CSS were $14.0 \pm 2.5$ and $17.0 \pm 2.6$ months, respectively, suggesting a poor prognosis for this cohort (Table 2).

\section{Univariate analyses of variables associated with OS or CSS in patients with metastatic chondrosarcoma}

Univariate analyses revealed that age, gender, race, location, and tumor type were not associated with either OS or CSS. Both OS and CSS differed significantly with tumor grade, with a high tumor grade portending a worse prognosis. Similarly, tumor size was associated with significant differences in OS and CSS, with tumor size $<10 \mathrm{~cm}$ predicting a better prognosis. In terms of treatment, patients who underwent surgical treatment had better OS and CSS than those who did not. However, radiation treatment had no significant effect on OS or CSS (Fig. 1A and C) and the addition of radiation treatment did not significantly improve the OS and CSS of patients who

underwent surgery (Fig. 1B and D). No obvious difference was observed in either OS or CSS between the radiation-only and no-therapy groups (Fig. 1B and D). A significant difference in OS but not CSS, based on tumor sequence was also observed (Table 2 and 3).

\section{Multivariate analysis of independent predictors of OS or CSS in patients with metastatic chondrosarcoma}

The multivariate analysis of all patients was shown in Table 4 . Tumor grade, tumor size, surgery for primary tumors, and tumor sequence were found to be independent predictors of OS. In terms of CSS, tumor grade, tumor size, and surgery were independent prognostic factors. The multivariate analysis showed no significant difference in either OS or CSS according to age and gender.

Table 2. Median survival data (month) of patients with primary chondrosarcoma of bone and metastatic disease at presentation

\begin{tabular}{|c|c|c|c|c|}
\hline Category & $\begin{array}{l}\text { OS } \\
\text { (estimate } \pm \\
\text { SE) }\end{array}$ & $95 \% \mathrm{CI}$ & $\begin{array}{l}\text { CSS (estimate } \pm \\
\text { SE) }\end{array}$ & $95 \% \mathrm{CI}$ \\
\hline Overall & $14.0 \pm 2.5$ & $9.1-18.9$ & $17.0 \pm 2.6$ & $11.9-22.1$ \\
\hline \multicolumn{5}{|l|}{ Age(years) } \\
\hline$<60$ & $14.0 \pm 3.1$ & $8.0-20.0$ & $14.0 \pm 3.0$ & $8.2-19.8$ \\
\hline$\geq 60$ & $18.0 \pm 4.6$ & $9.0-27.0$ & $25.0 \pm 4.9$ & $15.4-34.6$ \\
\hline \multicolumn{5}{|l|}{ Gender } \\
\hline Female & $9.0 \pm 3.0$ & $3.1-14.9$ & $11.0 \pm 3.9$ & $3.3-18.7$ \\
\hline Male & $19.0 \pm 2.7$ & $13.6-24.4$ & $20.0 \pm 5.7$ & 8.9-31.1 \\
\hline \multicolumn{5}{|l|}{ Race } \\
\hline White & $14.0 \pm 2.7$ & $8.6-19.4$ & $15.0 \pm 3.4$ & $8.4-21.6$ \\
\hline Other & $15.0 \pm 5.3$ & $4.7-25.3$ & $21.0 \pm 11.2$ & $0.0-42.9$ \\
\hline \multicolumn{5}{|l|}{ Location } \\
\hline Appendicular & $14.0 \pm 4.9$ & 4.4-23.6 & $21.0 \pm 4.1$ & $12.9-29.1$ \\
\hline Axial & $16.0 \pm 3.5$ & $9.2-22.8$ & $14.0 \pm 4.0$ & $6.2-21.8$ \\
\hline Rib, sternum and clavicle & $15.0 \pm 3.6$ & $8.0-22.0$ & $24.0 \pm 7.2$ & $9.8-38.2$ \\
\hline Other locations & $11.0 \pm 3.2$ & $4.8-17.2$ & $16.0 \pm 5.1$ & $6.1-25.9$ \\
\hline \multicolumn{5}{|l|}{ Tumor grade ${ }^{a}$} \\
\hline Low & $49.0 \pm 15.7$ & 18.2-79.8 & $66.0 \pm 29.7$ & $7.7-124.3$ \\
\hline High & $9.0 \pm 1.7$ & $5.6-12.4$ & $9.0 \pm 1.5$ & $6.1-11.9$ \\
\hline \multicolumn{5}{|l|}{ Tumor type } \\
\hline Chondrosarcoma, NOS & $14.0 \pm 2.9$ & 8.3-19.7 & $16.0 \pm 3.5$ & $9.2-22.8$ \\
\hline Other & $14.0 \pm 4.7$ & $4.8-23.2$ & $19.0 \pm 4.7$ & $9.7-28.3$ \\
\hline \multicolumn{5}{|l|}{ Tumor size } \\
\hline$<10 \mathrm{~cm}$ & $46.0 \pm 16.7$ & $13.3-78.7$ & 50.0 & $\mathrm{~N} / \mathrm{A}$ \\
\hline$\geq 10 \mathrm{~cm}$ & $11.0 \pm 2.2$ & $6.6-15.4$ & $13.0 \pm 2.3$ & $8.4-17.6$ \\
\hline \multicolumn{5}{|l|}{ Surgery } \\
\hline Yes & $29.0 \pm 5.0$ & $19.2-38.8$ & $30.0 \pm 7.8$ & $14.7-45.3$ \\
\hline No & $6.0 \pm 0.9$ & $4.2-7.8$ & $7.0 \pm 1.5$ & $4.1-9.9$ \\
\hline \multicolumn{5}{|l|}{ Radiation treatment } \\
\hline Yes & $12.0 \pm 4.2$ & $3.7-20.3$ & $14.0 \pm 3.9$ & $6.4-21.6$ \\
\hline No & $15.0 \pm 2.9$ & 9.4-20.6 & $19.0 \pm 4.5$ & $10.2-27.8$ \\
\hline \multicolumn{5}{|l|}{ Treatment modality } \\
\hline Surgery+radiation & $22.0 \pm 7.8$ & $6.8-37.2$ & $30.0 \pm 6.6$ & $17.1-42.9$ \\
\hline Surgery only & $30.0 \pm 10.3$ & $9.9-50.1$ & $31.0 \pm 14.6$ & $2.4-59.6$ \\
\hline Radiation only & $7.0 \pm 0.9$ & $5.2-8.8$ & $6.0 \pm 1.0$ & $4.0-8.0$ \\
\hline No therapy & $6.0 \pm 1.7$ & $2.6-9.4$ & $8.0 \pm 2.6$ & $2.9-13.1$ \\
\hline \multicolumn{5}{|l|}{ First cancer } \\
\hline Yes & $18.0 \pm 2.5$ & $13.1-22.9$ & $\mathrm{~N} / \mathrm{A}$ & $\mathrm{N} / \mathrm{A}$ \\
\hline No & $3.0 \pm 1.1$ & $0.9-5.1$ & $\mathrm{~N} / \mathrm{A}$ & $\mathrm{N} / \mathrm{A}$ \\
\hline
\end{tabular}



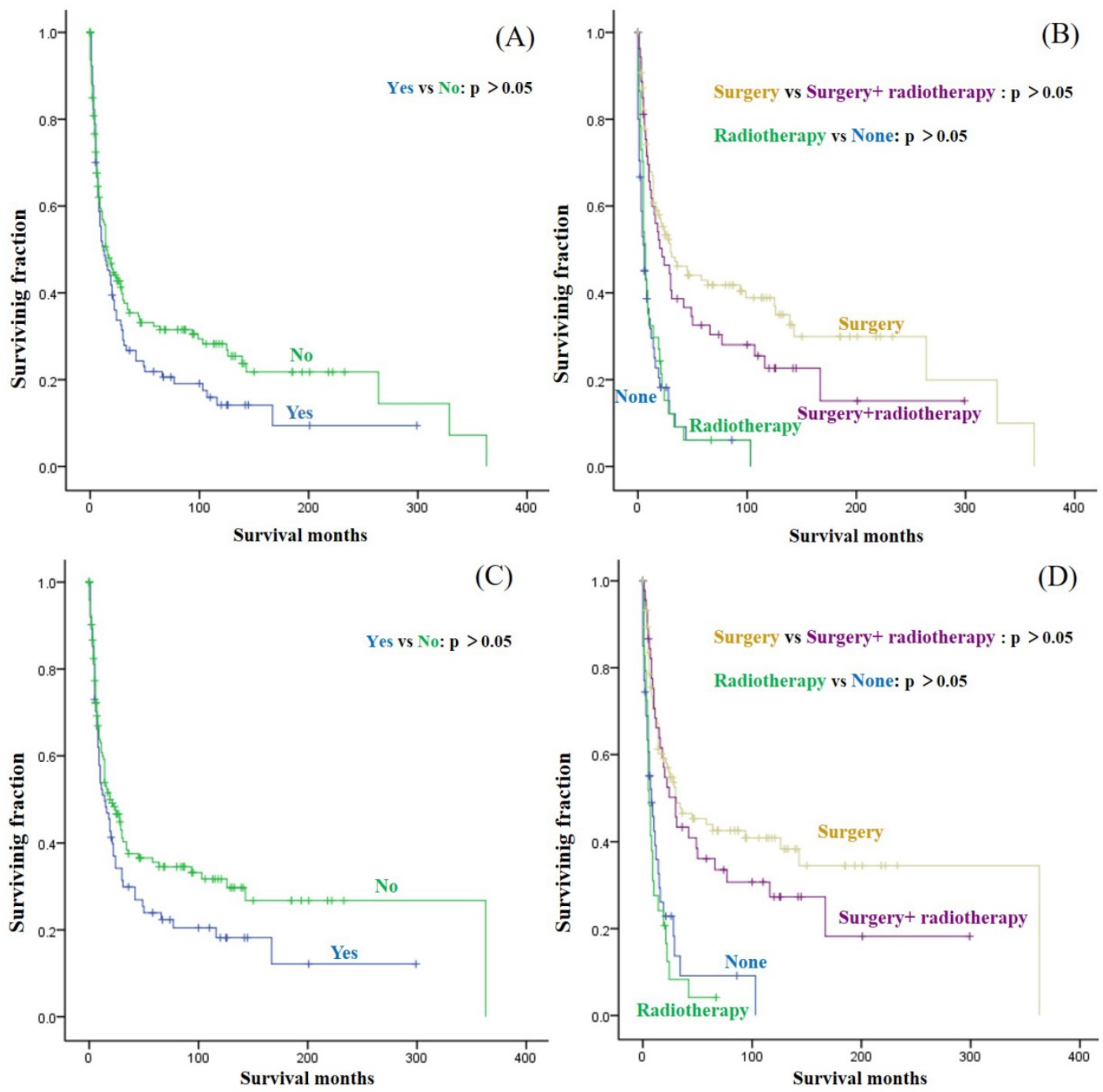

Figure 1. Kaplan-Meier method estimated OS in patients with primary chondrosarcoma of bone and metastatic disease at presentation stratified by (A) radiotherapy, (B) surgery + radiotherapy, and Kaplan-Meier estimated CSS in these patients stratified by (C) radiotherapy, (D) surgery + radiotherapy

\section{Discussion}

Chondrosarcoma is a malignancy of the mesenchymal tissue characterized by the production of cartilage matrix [13]. Although the majority of chondrosarcomas grow slowly and show late metastasis, the prognosis of patients with chondrosarcoma of bone and metastasis is very poor $[8,9,14]$. Because cases of primary chondrosarcoma of bone and metastatic disease at diagnosis are rare, few studies have documented the prognosis of metastatic chondrosarcoma [10, 14]. Our study is the first to describe the demographic information of such patients and explore possible predictors of survival using the SEER database.

The mean and median age at diagnosis of our cohort were 57.4 and 58 years, respectively, with a male predominance (1.6:1.0), similar to chondrosarcoma overall [4]. Chondrosarcoma not otherwise specified was the most common histological type (67.4\%), which was similar to a previous study of all chondrosarcoma [5]. Previous studies found that low-grade chondrosarcoma had a low rate of metastasis [15-18]. However, we found that metastasis at presentation was more frequent with low-grade tumors (42.4\%) than high-grade tumors $(29.9 \%)$, a result that requires further verification due to $27.7 \%$ unknown cases. The most common primary sites in our cohort were appendicular and pelvic sites, similar to all chondrosarcomas [1]. Damron et al. [3] reported that the 5-year survival rate for all chondrosarcoma was about $75.2 \%$ and Angelini et al. [19] reported that the survival rate of central conventional chondrosarcoma was $92 \%$ at 5 years and $84 \%$ at 10 years. Imai et al. [20] showed that the 5-year OS of unresectable chondrosarcomas was $53 \%$. By contrast, we found that the 5- and 10-year OS rates of the metastatic chondrosarcoma were $28.4 \%$ and $22.8 \%$, respectively, while the 5- and 10-year CSS rates were $31.2 \%$ and 
26.6\%. Additionally, the median OS and CSS of our cohort were $14.0 \pm 2.5$ months and $17.0 \pm 2.6$ months, respectively, suggesting a pretty poor prognosis. Because of the dismal survival of this patient population, it is necessary to explore prognostic factors to better guide the management of such patients.

Table 3. Univariate analysis of variables in patients with primary chondrosarcoma of bone and metastatic disease at presentation using Kaplan-Meier method

\begin{tabular}{lll}
\hline Variable & $\begin{array}{l}\text { OS (Log-rank p } \\
\text { value) }\end{array}$ & $\begin{array}{l}\text { CSS (Log-rank p } \\
\text { value) }\end{array}$ \\
\hline Age at diagnosis & 0.256 & 0.075 \\
Gender & 0.122 & 0.161 \\
Race & 0.656 & 0.262 \\
Location & 0.641 & 0.774 \\
Tumor grade (Low vs High) & $<0.001$ & $<0.001$ \\
Tumor type & 0.871 & 0.995 \\
Tumor size (<10cm vs $\geq 10 \mathrm{~cm})$ & $<0.001$ & $<0.001$ \\
Surgery (Yes vs No) & $<0.001$ & $<0.001$ \\
Radiation treatment (Yes vs No) & 0.119 & 0.089 \\
Treatment modality & $<0.001$ & $<0.001$ \\
Surgery+ radiotherapy vs Surgery & 0.185 & 0.361 \\
only & & \\
Surgery+ radiotherapy vs & $<0.001$ & $<0.001$ \\
Radiotherapy only & & $<0.001$ \\
Surgery+ radiotherapy vs No therapy & $<0.001$ & $<0.001$ \\
Surgery only vs Radiotherapy only & $<0.001$ & $<0.001$ \\
Surgery only vs No therapy & $<0.001$ & 0.527 \\
Radiotherapy only vs No therapy & 0.576 & 0.109 \\
First cancer & $<0.001$ & \\
\hline OS: overall survival; CSS: cancer-specific survival. a: Low: ICD-O-3 Grade 1 (well \\
differentiated) and Grade 2 (moderately differentiated); High: ICD-O-3 Grade 3 \\
(poorly differentiated) and Grade 4 (undifferentiated anaplastic).
\end{tabular}

Table 4. Multivariate analysis for OS and CSS for patients with primary chondrosarcoma of bone and metastatic disease at presentation

\begin{tabular}{lllll}
\hline Variable & OS & \multicolumn{2}{l}{ CSS } & \\
\hline & Hazard Ratio (95\% & P & Hazard Ratio (95\% & P \\
& value & CI) & value \\
\hline $\begin{array}{l}\text { Age at } \\
\text { diagnosis(years) }\end{array}$ & $0.997(0.989-1.005)$ & 0.453 & $0.995(0.987-1.004)$ & 0.253 \\
$\begin{array}{l}\text { Gender } \\
\text { Female }\end{array}$ & 1 & & & \\
$\begin{array}{l}\text { Male } \\
\text { Tumor grade }\end{array}$ & $0.973(0.722-1.313)$ & 0.859 & $0.953(0.681-1.334)$ & 0.780 \\
$\begin{array}{l}\text { Low } \\
\text { High }\end{array}$ & 1 & & & \\
$\begin{array}{l}\text { Tumor size } \\
<10 \mathrm{~cm}\end{array}$ & $2.497(1.742-3.581)$ & $<0.001$ & $2.544(1.689-3.830)$ & $<0.001$ \\
$\geq 10 \mathrm{~cm}$ & 1 & & & \\
$\begin{array}{l}\text { Surgery } \\
\text { Yes }\end{array}$ & $1.893(1.254-2.859)$ & 0.002 & $1.947(1.242-3.052)$ & 0.004 \\
No & 1 & & & \\
$\begin{array}{l}\text { First cancer } \\
\text { Yes }\end{array}$ & $2.465(1.785-3.404)$ & $<0.001$ & $2.647(1.856-3.773)$ & $<0.001$ \\
No & 1 & & & \\
\hline
\end{tabular}

OS: overall survival; CSS: cancer-specific survival. a: Low: ICD-O-3 Grade 1 (well differentiated) and Grade 2 (moderately differentiated); High: ICD-O-3 Grade 3 (poorly differentiated) and Grade 4 (undifferentiated anaplastic).

Univariate and multivariate analyses of this cohort showed that age was not associated with significant differences in either OS or CSS. The patients included in this study with short survival time may explain it. Giuffrida et al. [5] reported that an age of 50 years or less was associated with a significant OS benefit in chondrosarcoma, but was not an independent prognostic factor. Due to the improvement of diagnosis and treatment technology, only patients after 2000 were included in this study. We noted that race and gender were not associated with OS or CSS. An appendicular location of the chondrosarcoma was associated with a better prognosis compared with an axial location [5, 21, 22]. However, our univariate survival analysis revealed that tumor location was not significantly associated with either OS or CSS. The role of tumor site for metastatic chondrosarcoma needs to be clarified in the future. Tumor grade was usually recognized as a very important predictor of chondrosarcoma. Nakamura et al. [10] found that tumor grade was a significant predictor of prognosis in patients who developed lung metastases after the initial treatment of primary chondrosarcoma. Similarly, this study revealed that tumor grade could independently predict both OS and CSS of chondrosarcoma patients with metastasis at presentation. Therefore, tumor grade is an important prognostic factor for chondrosarcoma patients with metastasis, whether at presentation or otherwise.

Tumor type is of great significance in the prognosis of chondrosarcoma. In general, the prognosis of patients with dedifferentiated chondrosarcoma was reported to be much poorer than for other types, with a 5-year survival rate less $10 \%-25 \%$ [22-25]. Grimer et al. [26] reported that dedifferentiated chondrosarcoma patients with metastatic disease at presentation had extremely poor prognosis, with a median survival of 5 months. As chondrosarcoma, not otherwise specified (NOS) accounted for $67.4 \%$, other types were integrated for analysis. However, we found that tumor type made no difference to the prognosis, suggesting that less attention should be paid to tumor type in those metastatic patients. Many studies reported that the tumor size was associated with better survival of chondrosarcoma patients [21, 27]. Kamal et al. [7] found that tumor size was not correlated with survival and Bindiganavile et al. [22] revealed that tumor volume (in $\mathrm{mL}$ ) was not a predictor in chondrosarcoma. In our cohort, tumor size $>10 \mathrm{~cm}$ was an independent predictor of both OS and CSS.

Surgical excision is considered as the standard treatment for chondrosarcoma, but little is known regarding the standard treatment of patients with primary chondrosarcoma of bone and metastasis at presentation. Andreou et al. [14] found that metastatic chondrosarcoma patients who received further treatment, including chemotherapy, radiotherapy, 
and/or surgery, had significantly better survival than those receiving only supportive care. However, the effects of specific treatments on prognosis in their study were not clear. In our cohort, surgical resection of primary tumors significantly prolonged the survival of metastatic chondrosarcoma patients. In patients with metastasis, removing the primary tumor can alleviate pain, improve quality of life, and prolong survival [28]. Therefore, local surgery is an appropriate treatment for metastatic chondrosarcoma patients. Nakamura et al. [10] reported that treatment for metastases in chondrosarcoma patients also improved their survival. Regarding radiotherapy, a satisfactory outcome is usually not achieved in chondrosarcoma patients. Some recent studies reported that radiotherapy was associated with the prognosis in chondrosarcoma [6, 14, 29]. However, radiotherapy was not associated with survival in our study. The median OS and CSS of patients receiving radiotherapy tended to be lower than those receiving no radiotherapy, suggesting that radiation treatment was not beneficial for prolonging survival in this cohort.

This study had several limitations. First, the SEER database does not include information about local recurrence or metastasis during follow-up, which may affect the prognosis. Second, other known prognostic factors in cancer survival such as surgical method and chemotherapy, were not available in this database. Despite these shortcomings, the SEER database provides important insights into rare cancers, such as primary chondrosarcoma of bone and metastatic disease at diagnosis.

\section{Conclusion}

This is the largest population-based study to describe the demographics and analyze the prognosis for 264 patients with primary chondrosarcoma of bone and metastatic disease at diagnosis. OS and CSS rates of the entire group at 5-year were $28.4 \%$ and $31.2 \%$, respectively. The median OS and CSS were $14.0 \pm 2.5$ and $17.0 \pm 2.6$ months, respectively. Independent predictors of OS include tumor grade, tumor size, surgery and tumor sequence. Tumor grade, tumor size, and surgery were calculated as independent predictors of CSS. Our study explored OS and CSS and their risk factors in this special cohort and laid a solid foundation for future research of standard therapy.

\section{Acknowledgements}

This study was supported by the National Key R\&D Program of China (2018YFC1105400), and Medical and Health Science and Technology Plan of
Department of Health of Zhejiang Province (WKJ-ZJ-1821).

\section{Competing Interests}

The authors have declared that no competing interest exists.

\section{References}

1. Evola FR, Costarella L, Pavone V, Caff G, Cannavò L, Sessa A, et al. Biomarkers of Osteosarcoma, Chondrosarcoma, and Ewing Sarcoma. Frontiers in pharmacology. 2017; 8: 150 .

2. Sisu AM, Tatu FR, Stana LG, Petrescu CI, Tatu C, Motoc A. Chondrosarcoma of the upper end of the femur. Romanian journal of morphology and embryology $=$ Revue roumaine de morphologie et embryologie. 2011; 52: 709-13.

3. Damron TA, Ward WG, Stewart A. Osteosarcoma, chondrosarcoma, and Ewing's sarcoma: National Cancer Data Base Report. Clinical orthopaedics and related research. 2007; 459: 40-7.

4. Fiorenza F, Abudu A, Grimer RJ, Carter SR, Tillman RM, Ayoub K, et al. Risk factors for survival and local control in chondrosarcoma of bone. The Journal of bone and joint surgery British volume. 2002; 84: 93-9.

5. Giuffrida AY, Burgueno JE, Koniaris LG, Gutierrez JC, Duncan R, Scully SP. Chondrosarcoma in the United States (1973 to 2003): an analysis of 2890 cases from the SEER database. The Journal of bone and joint surgery American volume. 2009; 91: 1063-72.

6. van Maldegem AM, Gelderblom H, Palmerini E, Dijkstra SD, Gambarotti M, Ruggieri $\mathrm{P}$, et al. Outcome of advanced, unresectable conventional central chondrosarcoma. Cancer. 2014; 120: 3159-64.

7. Kamal AF, Husodo K, Prabowo Y, Hutagalung EU. Correlation between survival and tumour characteristics in patients with chondrosarcoma. Journal of orthopaedic surgery (Hong Kong). 2015; 23: 365-9.

8. Whelan JS, Davis LE. Osteosarcoma, Chondrosarcoma, and Chordoma. Journal of clinical oncology : official journal of the American Society of Clinical Oncology. 2018; 36: 188-93.

9. Speetjens FM, de Jong Y, Gelderblom H, Bovee JV. Molecular oncogenesis of chondrosarcoma: impact for targeted treatment. Current opinion in oncology. 2016; 28: 314-22.

10. Nakamura T, Matsumine A, Yamada S, Tsukushi S, Kawanami K, Ohno T, et al. Oncological outcome after lung metastasis in patients presenting with localized chondrosarcoma at extremities: Tokai Musculoskeletal Oncology Consortium study. OncoTargets and therapy. 2016; 9: 4747-51.

11. Goda JS, Ferguson PC, O'Sullivan B, Catton CN, Griffin AM, Wunder JS, et al. High-risk extracranial chondrosarcoma: long-term results of surgery and radiation therapy. Cancer. 2011; 117: 2513-9.

12. Kemmerer EJ, Gleeson E, Poli J, Ownbey RT, Brady LW, Bowne WB. Benefit of Radiotherapy in Extraskeletal Myxoid Chondrosarcoma: A Propensity Score Weighted Population-based Analysis of the SEER Database. American journal of clinical oncology. 2018; 41:674-80.

13. Polychronidou G, Karavasilis V, Pollack SM, Huang PH, Lee A, Jones RL. Novel therapeutic approaches in chondrosarcoma. Future oncology (London, England). 2017; 13: 637-48.

14. Andreou D, Ruppin S, Fehlberg S, Pink D, Werner M, Tunn PU. Survival and prognostic factors in chondrosarcoma: results in 115 patients with long-term follow-up. Acta orthopaedica. 2011; 82: 749-55.

15. Funovics PT, Panotopoulos J, Sabeti-Aschraf M, Abdolvahab F, Funovics JM, Lang S, et al. Low-grade chondrosarcoma of bone: experiences from the Vienna Bone and Soft Tissue Tumour Registry. International orthopaedics. 2011; 35: 1049-56.

16. Verdegaal SH, Brouwers HF, van Zwet EW, Hogendoorn PC, Taminiau AH. Low-grade chondrosarcoma of long bones treated with intralesional curettage followed by application of phenol, ethanol, and bone-grafting. The Journal of bone and joint surgery American volume. 2012; 94: 1201-7.

17. Andreou D, Gilg MM, Gosheger G, Werner M, Hardes J, Pink D, et al. Metastatic Potential of Grade I Chondrosarcoma of Bone: Results of a Multi-institutional Study. Annals of surgical oncology. 2016; 23: 120-5.

18. Eriksson AI, Schiller A, Mankin HJ. The management of chondrosarcoma of bone. Clinical orthopaedics and related research. 1980; (153):44-66.

19. Angelini A, Guerra G, Mavrogenis AF, Pala E, Picci P, Ruggieri P. Clinical outcome of central conventional chondrosarcoma. Journal of surgical oncology. 2012; 106: 929-37.

20. Imai R, Kamada T, Araki N. Clinical Efficacy of Carbon Ion Radiotherapy for Unresectable Chondrosarcomas. Anticancer research. 2017; 37: 6959-64.

21. Lee FY, Mankin HJ, Fondren G, Gebhardt MC, Springfield DS, Rosenberg AE, et al. Chondrosarcoma of bone: an assessment of outcome. The Journal of bone and joint surgery American volume. 1999; 81: 326-38.

22. Bindiganavile $\mathrm{S}$, Han I, Yun JY, Kim HS. Long-term Outcome of Chondrosarcoma: A Single Institutional Experience. Cancer research and treatment : official journal of Korean Cancer Association. 2015; 47: 897-903. 
23. Evans HL, Ayala AG, Romsdahl MM. Prognostic factors in chondrosarcoma of bone: a clinicopathologic analysis with emphasis on histologic grading. Cancer. 1977; 40: 818-31.

24. Mercuri M, Picci P, Campanacci L, Rulli E. Dedifferentiated chondrosarcoma. Skeletal radiology. 1995; 24: 409-16.

25. Italiano A, Mir O, Cioffi A, Palmerini E, Piperno-Neumann S, Perrin C, et al. Advanced chondrosarcomas: role of chemotherapy and survival. Annals of oncology : official journal of the European Society for Medical Oncology. 2013; 24: 2916-22.

26. Grimer RJ, Gosheger G, Taminiau A, Biau D, Matejovsky Z, Kollender Y, et al. Dedifferentiated chondrosarcoma: prognostic factors and outcome from a European group. European journal of cancer (Oxford, England : 1990). 2007; 43: 2060-5.

27. Roos E, van Coevorden F, Verhoef C, Wouters MW, Kroon HM, Hogendoorn PC, et al. Prognosis of Primary and Recurrent Chondrosarcoma of the Rib. Annals of surgical oncology. 2016; 23: 811-7.

28. Tao L, Yuan C, Ma Z, Jiang B, Xiu D. Surgical resection of a primary tumor improves survival of metastatic pancreatic cancer: a population-based study. Cancer management and research. 2017; 9: 471-9.

29. Arshi A, Sharim J, Park DY, Park HY, Bernthal NM, Yazdanshenas H, et al. Chondrosarcoma of the Osseous Spine: An Analysis of Epidemiology, Patient Outcomes, and Prognostic Factors Using the SEER Registry From 1973 to 2012. Spine. 2017; 42: 644-52 